\title{
Reducing Memory Interference in Multicore Systems via Application-Aware Memory Channel Partitioning
}

\author{
SAFARI Technical Report No. 2011-002 \\ June 3, 2011
}

\author{
Sai Prashanth Muralidhara \\ Pennsylvania State University \\ smuralid@cse.psu.edu \\ Onur Mutlu \\ Carnegie Mellon University \\ onurecmu.edu
}

\author{
Lavanya Subramanian \\ Carnegie Mellon University \\ lsubrama@andrew. cmu . edu \\ Mahmut Kandemir \\ Pennsylvania State University \\ kandemir@cse.psu.edu
}

Thomas Moscibroda

Microsoft Research

moscitho@microsoft.com

\begin{abstract}
Main memory is a major shared resource among cores in a multicore system. If the interference between different applications' memory requests is not controlled effectively, system performance can degrade significantly. Previous work aimed to mitigate the problem of interference between applications by changing the scheduling policy in the memory controller, i.e., by prioritizing memory requests from applications in a way that benefits system performance.

In this paper, we first present an alternative approach to reducing inter-application interference in the memory system: application-aware memory channel partitioning (MCP). The idea is to map the data of applications that are likely to harmfully interfere with each other to different memory channels. The key principles are to partition the data of 1) light (memory non-intensive) and heavy (memory intensive) applications, and of 2) applications with low and high row-buffer locality onto separate channels, respectively. We show that by doing so, averaged over 240 workloads on a 24-core system with 4 memory channels, MCP improves system throughput by $7.1 \%$ over an application-unaware memory scheduler and by $1 \%$ over the best previous scheduler, while avoiding modifications to existing memory schedulers.

Second, we observe that interference can be even further reduced with a combination of MCP and memory scheduling, which we call integrated memory partitioning and scheduling (IMPS). The key idea is to 1) always prioritize very light applications in the memory scheduler since such applications cause negligible interference to others, 2) use memory channel partitioning to reduce interference between the remaining applications. Extensive evaluations on a variety of multi-programmed workloads and
\end{abstract}


system configurations show that this integrated memory partitioning and scheduling approach provides better system performance than MCP and four previous memory scheduling algorithms employed alone. Averaged over 240 workloads on a 24-core system with 4 memory channels, IMPS improves system throughput by $11.1 \%$ over an application unaware scheduler and $5 \%$ over the current best scheduling policy, while incurring much lower hardware complexity than the latter.

\section{Introduction}

Applications executing concurrently on a multicore chip contend with each other to access main memory, which has limited bandwidth. If the limited memory bandwidth is not managed well, different applications can harmfully interfere with each other, which can result in significant degradation in both system performance and individual application performance $[10,11,15,16,17,19]$. Several techniques to improve system performance by reducing memory interference among applications have been proposed $[10,11,15,16,17,19,21]$. Fundamentally, these proposals viewed the problem as a memory access scheduling problem, and consequently focused on developing new memory request scheduling policies that prioritize the requests of different applications in a way that reduces inter-application interference. However, such application-aware scheduling algorithms require (non-negligible) changes to the existing memory controllers' scheduling logic $[11,28]$.

In this paper, we present and explore a fundamentally-different alternative approach to reducing interapplication interference in the memory system: controlling the mapping of applications' data to memory channels. Our approach is based on the observation that multicore systems have multiple main memory channels $[2,4,10]$ each of which controls a disjoint portion of physical memory and can be accessed independently without any interference [10]. This reveals an interesting trade-off. On the one hand, interference between applications could (theoretically) be completely eliminated if each application's accesses were mapped to a different channel, assuming there were enough channels in the system. But, on the other hand, even if so many channels were available, mapping each application to its own channel would under utilize memory bandwidth and capacity (some applications may need less bandwidth/capacity than they are assigned, while others need more) and would reduce the opportunity for bank/channel-level parallelism within each application's memory access stream. Therefore, the main idea of our approach is to find a sweet spot in this trade-off by mapping the data (i.e., memory pages) of applications that are likely to cause significant interference/slowdown to each other to different memory channels.

We make two major contributions. First, we explore the potential of reducing inter-application memory interference purely with channel partitioning, without modifying the memory scheduler. To this end, we develop a new Application-Aware Memory Channel Partitioning (MCP) algorithm that assigns preferred memory channels to different applications. The goal is to assign any two applications whose mutual interference would cause significant slowdowns, to different channels. Our algorithm operates using a set of heuristics which are guided by insight about how applications with different memory access characteristics interfere with each other. Specifically, we show in Sec 3 that, whenever possible, applications of largely divergent memory-intensity or row-buffer-hit rate should be separated onto different channels.

Second, we show that MCP and traditional memory scheduling approaches are orthogonal in the sense that both concepts can beneficially be applied together. Specifically, whereas our MCP algorithm is agnostic to the memory scheduler (i.e., we assume an unmodified, commonly used row-hit-first memory 
scheduler [22, 30]), we show that additional gains are possible when using MCP in combination with a minimal-complexity application-aware memory scheduling policy. We develop an Integrated Memory Partitioning and Scheduling (IMPS) algorithm that seamlessly divides the work of reducing interapplication interference between the memory scheduler and the system software's page mapper based on what each can do best.

The key insight underlying the design of IMPS is that interference suffered by very low memoryintensity applications is more easily mitigated by prioritizing them in the memory scheduler, than with channel partitioning. Since such applications seldom generate requests, prioritizing their requests does not cause significant interference to other applications, as previous work has also observed [10, 11]. Furthermore, explicitly allocating one or more channels for such applications can result in a waste of bandwidth. Therefore, IMPS prioritizes requests from such applications in the memory scheduler, without assigning them dedicated channels, while reducing interference between all other applications using channel partitioning.

Overview of Results: We evaluate MCP and IMPS on a wide variety of multi-programmed applications and systems and in comparison to a variety of pure memory scheduling algorithms. Our first main finding is that on a 24-core 4-memory controller system with an existing application-unaware memory scheduler, MCP provides slightly higher performance benefits than the best previous memory scheduling algorithm, Thread Cluster Memory Scheduling (TCM) [11]: 7.1\% performance improvement vs. $6.1 \%$ for TCM. This performance improvement is achieved with no modification to the underlying scheduling policy. Furthermore, we find that IMPS provides better system performance than current state-of-the-art memory scheduling policies, pure MCP, as well as combinations of MCP and state-of-the-art scheduling policies: 5\% over the best scheduler, while requiring smaller hardware complexity.

Our main conclusion is that the task of reducing harmful inter-application memory interference should be divided between the memory scheduler and the system software page mapper. Only the respective contributions of both entities yields the best system performance.

\section{Background}

We present a brief background about the DRAM main memory system; more details can be found in $[6,16,22]$. A modern main memory system consists of several channels. Each channel can be accessed independently, i.e., accesses to different channels can proceed in parallel. A channel, in turn, is organized as several banks. These banks can be accessed in parallel; however, the data and address buses are shared among the banks, and data from only one bank can be sent through the channel at any time.

Each DRAM bank has a 2D structure consisting of rows and columns. A column is the smallest addressable unit of memory, and a large number of columns make up a row. When a unit of data has to be accessed from a bank, the row containing the data is brought into a small internal buffer called the row buffer. If subsequent memory access requests are to the same row, they can be serviced faster (2-3 times) than accessing a new row. This is called a row-hit. In order to improve DRAM data throughput, modern memory controller scheduling algorithms prioritize row-hits over row-misses.

Memory Request Scheduling Policy. FR-FCFS [22,30] is a commonly used scheduling policy in current commodity systems. It prioritizes row-hits over row-misses, and within each category, it prioritizes older requests. The analyses in this paper assume the FR-FCFS scheduling policy, but our insights are applicable to other scheduling policies as well. Sec 7 describes other memory scheduling policies and Sec 8 qualitatively and quantitatively compares our approach to them.

OS Page Mapping Policy. The Operating System (OS) maps a virtual address to a physical address. 
The address interleaving policy implemented in the memory controller in turn maps the physical address to a specific channel/bank in the main memory. Row interleaving and cache line interleaving are two commonly used interleaving policies. In the row interleaving policy, consecutive rows of memory are mapped to consecutive memory channels. We assume equal sizes for OS pages and DRAM rows in this work and use the terms page and row interchangeably without loss of generality. ${ }^{1}$ Pure cache line interleaving maps consecutive cache lines in physical address space to consecutive memory channels. MCP cannot be applied on top of this, as a page has to stay within a channel for MCP. However, we can potentially apply MCP on top of a restricted version of cache line interleaving that maps consecutive cache lines of a page to banks within a channel.

Commonly used OS page mapping and address interleaving policies are application-unaware and map applications' pages across different channels. The OS does not consider inter-application interference and channel information while mapping a virtual page to a physical page. It simply uses the next physical page to allocate/replace based on recency of use. We build our discussions, insights and mechanisms assuming such an interference-unaware OS page mapping policy and a row interleaved address mapping policy. However, we also evaluate MCP on top of cache line interleaving across banks in Sec 9.4.

Memory Related Application Characteristics. We characterize memory access behavior of applications using two attributes. Memory Access Intensity is defined as the rate at which an application misses in the last level on-chip cache and accesses memory - calculated as Misses per Kilo Instructions (MPKI). Row Buffer Locality is defined as the fraction of an application's accesses that hit in the row buffer (i.e., access to an open row). This is calculated as the average Row-Buffer Hit Rate (RBH) across all banks.

\section{Motivation}

In this section, we motivate our partitioning approach by showing how applications with certain characteristics cause more interference to other applications, and how careful mapping of application pages to memory channels can ameliorate this problem.



(a) Conventional Page Mapping.

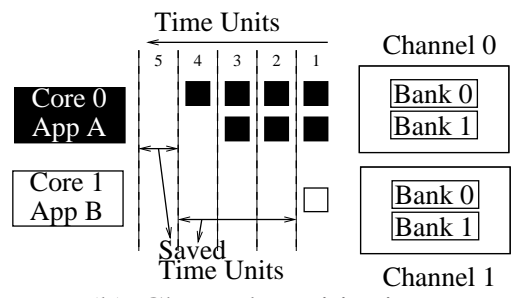

(b) Channel Partitioning.

Figure 1. Conceptual example showing benefits of mapping data of low and high memory-intensity applications to separate channels.

In Figure 1, we present a conceptual example showing the performance benefits of mapping the pages of applications with largely different memory-intensities to separate channels. Application $A$ on Core 0 has high memory-intensity, generating memory requests at a high rate; Application $B$ on Core 1 has low memory-intensity and generates requests at a much lower rate. Figures 1(a) and 1(b) show characteristic examples of what can happen with conventional page mapping (where the pages of $A$ and $B$ are mapped to the same channels) and with application-aware channel partitioning (where $A$ and $B$ 's pages are mapped to separate channels), respectively. In the first case, $B$ 's single request is queued up behind 3 of

\footnotetext{
${ }^{1}$ Our mechanism works as long as the row size is greater than the OS page size, as is the case in typical systems.
} 
$A$ 's requests in a bank of Channel 0 (see Fig 1(a)). As a result, Application $B$ stalls for a long period of time (4 DRAM bank access latencies, in this example) until the 3 previously scheduled requests from $A$ to the same bank get serviced. In contrast, if the two applications' data are mapped to separate channels as shown in Figure 1(b), $B$ 's request is not queued and can be serviced immediately, leading to $B$ 's fast progress ( 1 access latency vs 4 access latencies). Furthermore, even Application $A$ 's access latency improves ( 4 vs. 5 time units) because the interference caused to it by $B$ 's single request is eliminated.

To determine to what extent such effects occur in practice, we ran a large number of simulation experiments ${ }^{2}$ with applications of vastly different memory-intensities and present a representative result: We run four copies each of milc and $h 264$ (from the SPEC CPU2006 suite [1]) on an eight-core, two-channel system. Figure 2 shows the effects of conventional channel sharing: $h 264$, the application with lower memory-intensity, is slowed down by $2.7 \mathrm{x}$ when sharing memory channels with milc. On the other hand, if milc's and h264's data are partitioned and mapped to Channels 0 and 1 , respectively, $h 264$ 's slowdown reduces to $1.5 x$. Furthermore, milc's slowdown also drops from $2.3 \mathrm{x}$ to $2.1 \mathrm{x}$, as its queue-

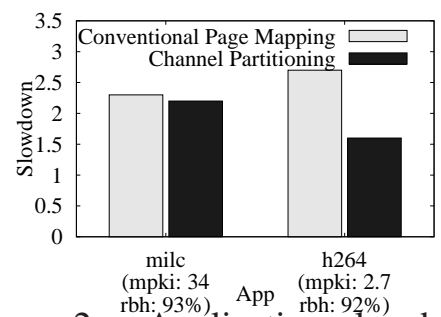

Figure 2. Application slowdowns due to interference between high and low memory-intensity applications. ing delays reduce due to reduced interference from $h 264$. This substantiates our intuition from the example: Separating the data of low memory-intensity applications from that of the high memory-intensity applications can largely improve the performance of both the low memory-intensity applications and the overall system.

Memory-intensity is not the only characteristic that determines the relative harmfulness of applications. In Figure 3, we show potential benefits of mapping memory-intensive applications with significantly different row-buffer localities onto separate channels. In the example, Application $A$ accesses the same row, Row 5, repeatedly and hence has much higher row-buffer locality than Application $B$, whose accesses are to different rows, incurring many row misses.

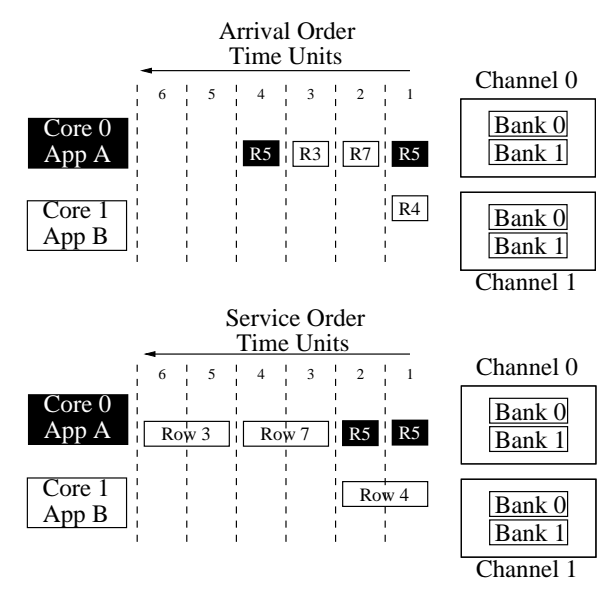

(a) Conventional Page Mapping

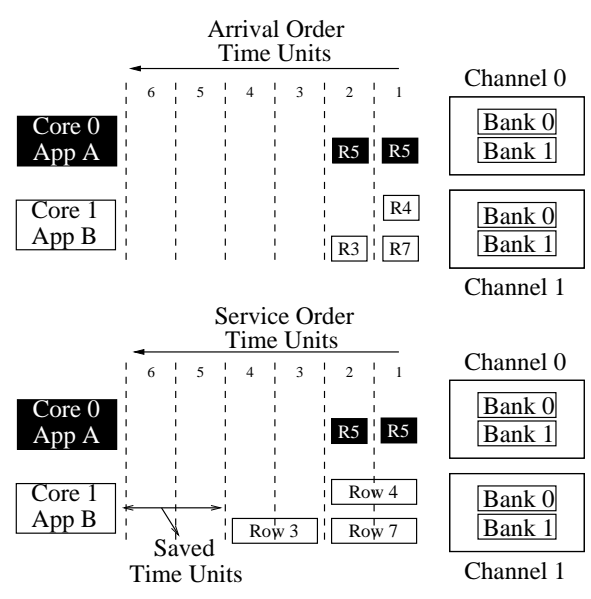

(b) Channel Partitioning

Figure 3. Conceptual example showing benefits of mapping data of low and high row-buffer hit rate memoryintensive applications to separate channels. In both (a) and (b), the top part shows the request arrival order and the bottom part shows the order in which the requests are serviced.

\footnotetext{
${ }^{2}$ Our simulation methodology is described in Sec 8 .
} 
Figure 3(a) shows a conventional page mapping approach, while Figure 3(b) shows a channel partitioning approach. With conventional mapping, the commonly used row-hit-first memory scheduling policy prioritizes $A$ 's requests over $B$ 's requests to Rows 7 and 3, even though $B$ 's requests had arrived earlier (Figure 3(a)). This leads to increased queueing delays of $B$ 's requests causing $B$ to slow down. On the other hand, if the pages of $A$ and $B$ are mapped to separate channels (Figure 3(b)), the interference received by $B$ is reduced and consequently the queueing delays experienced by $B$ 's requests reduced (by 2 time units). This improves Application $B$ 's performance without affecting Application A's.

A representative case study from among our experiments is shown in Figure 4. We ran four copies each of mcf and libquantum, two memory-intensive applications on an eight-core twochannel system. Mcf has a low row-buffer hit rate of $42 \%$ and suffers a slow down of $20.7 \mathrm{x}$ when sharing memory channels with libquantum, which is a streaming application with $99 \%$ row-buffer hit rate. On the other hand, if the data of $m c f$ is isolated from libquantum's data and given a separate channel, mcf's slowdown drops significantly, to $6.5 x$ from $20.7 x$. Libquantum's small performance loss of $4 \%$ shows the trade-off involved in channel partitioning: The drop is due to the loss in bank-level parallelism resulting from assigning only one channel to libquantum. In terms

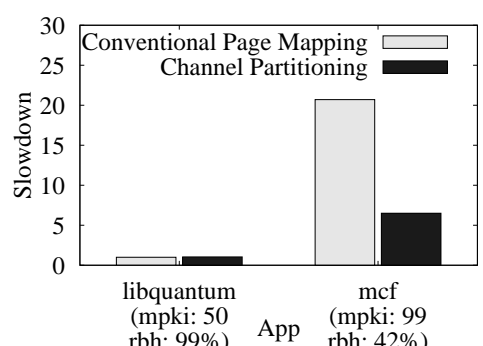

Figure 4. ${ }^{\text {rbh: } 99 \%)}$ Application shlowdowns due to interference between high and low row-buffer hit rate memoryintensive applications. of system performance, however, this drop is far outweighed by the reduction in slowdown of $m c f$. We therefore conclude that isolating applications with low row-buffer locality from applications with high row-buffer locality by means of channel partitioning improves the performance of applications with low row-buffer locality and the overall system.

Based on these insights, we next develop MCP, an OS-level mechanism to partition the main memory bandwidth across the different applications running on a system. Then, we examine how to best combine memory partitioning and scheduling to minimize inter-application interference and obtain better system performance.

\section{Memory Channel Partitioning Mechanism (MCP)}

Our MCP mechanism consists of three components: 1) profiling of application behavior during run time, 2) assignment of preferred channels to applications, 3) allocation of pages to the preferred channel. The mechanism proceeds in periodic intervals. During each interval, application behavior is profiled (Sec 4.1). At the end of an interval, the applications are categorized into groups based on the characteristics collected during the interval, and each application is accordingly assigned a preferred channel (Sec 4.2). In the subsequent interval, these preferred channel assignments are applied. That is, when an application accesses a new page that is either not currently in DRAM or not in the application's preferred channel, MCP uses the preferred channel assignment for that application: The requested page is allocated in the preferred channel, or migrated to the preferred channel (see Sec 4.3).

In summary, during the $X$ th interval, MCP applies the preferred channel assignment which was computed at the end of the $(X-1)$ st interval, and also collects statistics, which will then be used to compute the new preferred channel assignment to be applied during the $(X+1)$ st execution interval. ${ }^{3}$ Note that

\footnotetext{
${ }^{3}$ The very first interval is used for profiling only. We envision it to be shorter than the subsequent execution intervals,
} 
MCP does not constrain the memory usage of applications. It provides a preferred channel assignment in order to reduce interference. Therefore, applications can use the entire DRAM capacity, if needed.

\subsection{Profiling of Application Characteristics}

As shown in Sec 3, memory access intensity and row-buffer locality are key factors determining the level of harm caused by interference between applications. Therefore, during every execution interval, each application's Misses Per Kilo Instruction (MPKI) and Row-Buffer Hit Rate (RBH) statistics are collected. To compute an application's inherent row-buffer hit rate, we use a per-core shadow rowbuffer index for each bank, as in previous work $[8,11,16]$, which keeps track of the row that would have been present in the row-buffer had the application been running alone.

\subsection{Preferred Channel Assignment}

At the end of every execution interval, each application is assigned a preferred channel. The assignment algorithm is based on the insights derived in Sec 3. The goal is to separate as much as possible 1) the data of low memory-intensity applications from that of high memory-intensity applications, and, 2) among the memory-intensive applications, the data of low row-buffer locality applications from that of high row-buffer locality applications. To do so, MCP executes the following steps in order:

1. Categorize applications into low and high memory-intensity groups based on their MPKI. (Sec 4.2.1) 2. Further categorize the high memory-intensity applications, based on their row-buffer hit rate $(\mathrm{RBH})$ values into low and high row-buffer hit rate groups. (Sec 4.2.2)

3. Partition the available memory channels among the three application groups. (Sec 4.2.3)

4. For each application group, partition the set of channels allocated to this group between all the applications in that group, and assign a preferred channel to each application. (Sec 4.3)

\subsubsection{Intensity Based Grouping}

MCP categorizes applications into low and high memory-intensity groups based on a threshold parameter, $M P K I_{t}$. MPKI $I_{t}$ is determined by averaging the last level cache MPKI of all applications and multiplying it by a scaling factor. For every application $i$, if its MPKI, $M P K I_{i}$ is less than $M P K I_{t}$, the application is categorized as low memory-intensity, else high memory-intensity. The average value of MPKI provides a threshold that adapts to the workload's memory intensity and acts as a separation point in the middle of the workload's memory-intensity range. The scaling factor further helps to move this point up or down the range, regulating the number of applications in the low memory-intensity group. We empirically found that a scaling factor of 1 provides an effective separation point and good system performance (Sec 9).

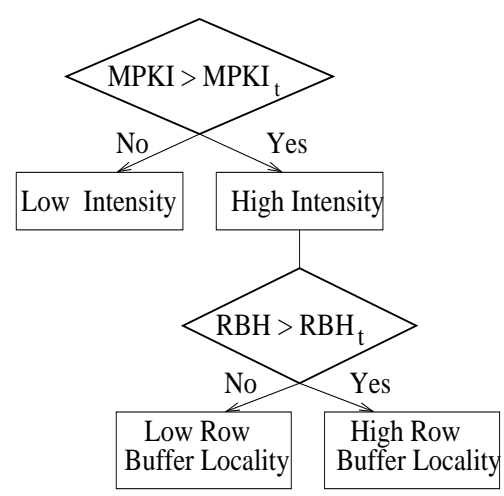

Figure 5. MCP: Application Grouping.

and its length is a trade off between minimizing the number of pages that get allocated before the first set of channel preferences are established and letting the application's memory access behavior stabilize before collecting statistics. (Empirical evaluation in Sec 9.6) 


\subsubsection{Row-Buffer Locality Based Grouping}

MCP further classifies the high memory-intensity applications into either low or high row-buffer locality groups based on a threshold parameter, $R B H_{t}$. For every application $i$, if its $R B H_{i}$ is less than $R B H_{t}$, then it is classified as a low row-buffer locality application. In this case, we do not take an average or use a scaling factor, as we observe that inter-application interference due to row-buffer locality differences are more pronounced between applications with very low and high row-buffer localities, unlike memoryintensity where there is interference across the continuum. We empirically observe that an $R B H_{t}$ value of $50 \%$ provides effective interference reduction and good performance (Sec 9).

\subsubsection{Partitioning of Channels between Application Groups}

After thus categorizing applications into 3 groups, MCP partitions the available memory channels between the groups. It is important to note that at this stage of the algorithm, memory channels are assigned to application groups and not to individual applications. MCP handles the preferred channel assignment to individual applications in the next step (Sec 4.2.4). Channels are first partitioned between low and high memory-intensity groups. The main question is how many channels should be assigned to each group. One possibility is to allocate channels proportional to the total bandwidth demand (sum of applications' MPKI) of each group (bandwidth proportional allocation). This amounts to balancing the total bandwidth demand across channels. Alternatively, channels could be allocated proportional to the number of applications in that group (application count proportional allocation). In the former case, the low memory-intensity applications which constitute a very low proportion of total bandwidth demand might be assigned no channels. This fails to achieve their isolation from high memory-intensity applications, leading to low system performance. In contrast, if the latter is used, it results in bandwidth wastage as the low memory-intensity applications seldom generate requests and the bandwidth of the channels they are assigned to would have been better utilized by the high memory-intensity applications. We found that the isolation benefits of application-count-proportional allocation outweighs the bandwidth wastage caused by potentially allocating low-intensity applications to one or more channels. ${ }^{4}$ Therefore, we use the application count proportional channel allocation strategy for MCP. However, bandwidth wastage caused by potentially allocating very low intensity applications dedicated channels remains, and we will show that eliminating this wastage by handling these applications in the scheduler in an integrated scheduling and partitioning mechanism is beneficial ( $\operatorname{Sec} 5$ ). The channels allocated to the high memory-intensity group are further partitioned between the low and high row-buffer locality groups. The applications in the high memory-intensity group are bandwidth sensitive, meaning they each need a fair share of bandwidth to make progress. To ensure this, MCP assigns a number of channels to each of these two groups proportionally to the bandwidth demand (sum of MPKIs) of the group.

\subsubsection{Preferred Channel Assignment within an Application Group}

As a final step, MCP determines which applications within a group are mapped to which channels, when more than one channel is allocated to a group. Within each group, we balance the total bandwidth demand across the allocated channels. For each group, we maintain a ranking of applications by memory-intensity. We start with the least intensive application in the group and map applications to

\footnotetext{
${ }^{4} \mathrm{We}$ found that bandwidth proportional allocation results in a $4 \%$ performance loss over the baseline since it increases memory interference.
} 
the group's first allocated channel until the bandwidth demand allocated to it (approximated by sum of $M P K I_{i}$ of every application $i$ allocated to it) is $\frac{\text { Sum of MPKIs of applications in the group }}{\text { Number of channels allocated to the group }}$ We then move on to the next channel and allocate applications to it. This is repeated for every application group. At the end of this procedure, each application is assigned a preferred channel.

\subsection{Allocation of Pages to Preferred Channel}

Once each application is assigned a preferred channel, MCP allocates a page to the preferred channel in case it is not already there. There are two possibilities. First, a page fault: the accessed page is not present in any channel. In this case, the page fault handler attempts to allocate the page in the preferred channel. If there is a free page in the preferred channel, the new page is allocated there. Otherwise, a modified version of the CLOCK replacement policy, as described in [7] is used. The baseline CLOCK policy keeps a circular list of pages in memory, with the hand (iterator) pointing to the oldest allocated page in the list. There is a Referenced (R) bit for each page, that is set to ' 1 ' when the page is referenced. The $\mathrm{R}$ bits of all pages are cleared periodically by the operating system. When a page fault occurs and there are no free pages, the hand moves over the circular list until an unreferenced page (a page with $R$ bit set to ' 0 ') is found. The goal is to choose the first unreferenced page as the replacement. To allocate a page in the preferred channel, the modified CLOCK algorithm looks ahead $\mathrm{N}$ pages beyond the first replacement candidate to potentially find an unreferenced page in the preferred channel. If there is no unreferenced page within $\mathrm{N}$, the first unreferenced page in the list across all channels is chosen as the replacement candidate. We use an $\mathrm{N}$ value of 512 .

Second, the accessed page is present in a channel other than the preferred channel, which we observe to be very rare in our workloads, since application behavior is relatively constant within an interval. In this case, dynamically migrating the page to the preferred channel could be beneficial. However, dynamic page migration incurs TLB and cache block invalidation overheads as discussed in [3]. We find that less than $12 \%$ of pages in all our workloads go to non-preferred channels and hence migration does not gain much performance over allowing some pages of an application to potentially remain in the non-preferred channels. Thus, our default implementation of MCP does not do migrations. However, if needed, migration can of course be seamlessly incorporated into MCP and IMPS.

\section{Integrated Memory Partitioning and Scheduling (IMPS)}

MCP aims to solve the inter-application memory interference problem entirely with the system software's page mapper (with the support of additional hardware counters to collect MPKI and RBH metrics for each application). It does not require any changes to the memory scheduling policy. This approach is in stark contrast to the various existing proposals, which try to solve the problem "from the opposite side". These proposals aim to reduce memory interference entirely in the memory controller hardware using sophisticated scheduling policies (e.g., $[10,11,16,17])$ The question is whether either extreme alone (i.e., page mapping alone and memory scheduling alone) can really provide the best possible interference reduction. Based on our observations, the answer is negative. Specifically, we devise an integrated memory partitioning and scheduling (IMPS) mechanism that aims to combine the interference reduction benefits of both.

The key observation underlying IMPS is that applications with very low memory-intensity, when prioritized over other applications in the memory scheduler, do not cause significant slowdowns to other applications. This observation was also made in previous work $[10,11]$. These applications seldom generate memory requests; prioritizing these requests enables the applications to quickly continue with 
long computation periods and utilize their cores better, thereby significantly improving system throughput $[10,11]$. As such, scheduling can very efficiently reduce interference that affects very low memoryintensity applications. In contrast, reducing the interference against such applications purely using the page mapper is inefficient. The mapper would have to dedicate one or more channels to such lowmemory-intensity applications, wasting memory bandwidth, since these applications do not require significant memory bandwidth (yet high-intensity applications would likely need the wasted bandwidth, but cannot use it). If the mapper cannot dedicate a channel to such applications, they would share channels with high-intensity applications and experience high interference with an unmodified memory scheduler.

The basic idea and operation of IMPS is therefore simple. First, identify at the end of an execution interval very low memory-intensity applications (i.e., applications whose MPKI is smaller than a very low threshold, 1.5 in most of our experiments (Sec 9.6)), prioritize them in the memory scheduler over all other applications in the next interval, and allow the mapping of the pages of such applications to any memory channel. Second, reduce interference between all other applications by using memory channel partitioning (MCP), exactly as described in Sec 4. The modification to the memory scheduler is minimal: the scheduler only distinguishes the requests of very low memory-intensity applications over those of others, but does not distinguish between requests of individual applications in either group. The memory scheduling policy consists of three prioritization rules: 1) prioritize requests of very low memory-intensity applications, 2) prioritize row-hit-first requests, 3) prioritize older requests.

Note that MCP is still used to classify the remaining applications as low and high memory-intensity, as only the very low memory-intensity applications are filtered out and prioritized in the scheduler. MCP's channel partitioning still reduces interference and consequent slowdowns of the remaining applications.

\section{Implementation}

Hardware support. MCP requires hardware support to estimate MPKI and row-buffer hit rate of each application, as described in Sec 4.1. These counters are readable by the system software via special instructions. Table 1 shows the storage cost incurred for this purpose. For a 24-core system with 4 memory controllers (each controlling 4 memory banks and 16384 rows per bank), the hardware overhead is $12 \mathrm{~K}$ bits. IMPS requires an additional bit per each request (called low-intensity bit) to distinguish very low-memory-intensity applications' requests over others, which is an additional overhead of only 512 bits for a request queue size of 128 per MC. IMPS also requires small modifications to the memory scheduler to take into account the low-intensity bits in prioritization decisions. Note that, unlike previous application-aware memory request scheduling policies, IMPS (or MCP) 1) does not require each main memory request to be tagged with a thread/application ID since it does not distinguish between individual applications' requests, 2) adds only a single new bit per request for the memory scheduler to consider, 3) does not require application ranking as in [10,11,17] - ranking and prioritization require hardware logic for sorting and performing comparisons. As such, the complexity of IMPS is much lower than previous application-aware memory scheduling policies.

System software support. MCP and IMPS require support from system software to 1) read the counters provided by the hardware, 2) perform the preferred channel assignment, at the end of each execution interval, as already described. Each application's preferred channel is stored as part of the system software's data structures, leading to a very modest memory overhead of $N_{\text {AppsInSystem }} \times N_{\text {MemoryChannels }}$. The page fault handler and the page replacement policy are modified slightly, as described in Sec 4.3. Our experiments show that the execution time overheads of the required tasks are negligible. Note that our proposed mechanisms do not require changes to the page table. 
SAFARI Technical Report No. 2011-002 (June 3, 2011)

\begin{tabular}{|l|l|l|}
\hline Storage & Description & Size \\
\hline \hline Storage Overhead for MCP - per-core registers & $N_{\text {core }} \times \log _{2} M P K I_{m a x}=240$ \\
\hline MPKI-counter & A cores last level cache misses per kilo instruction & $N_{\text {core }} \times N_{\text {banks }} \times \log _{2} N_{\text {rows }}=1344$ \\
\hline Storage Overhead for MCP - per-core registers in each controller & $N_{\text {core }} \times N_{\text {banks }} \times \log _{2} C o u n t_{m a x}=1536$ \\
\hline Shadow row-buffers & Row address of a core's last accessed row \\
\hline Shadow row-buffer hit counters & Number of row-hits if the application were running alone \\
\hline \hline Additional Storage Overhead for IMPS - per request register in each controller & $1 \times Q u e u e_{\max }=128$ \\
\hline Very low memory-intensity indicator & To identify requests from very low memory-intensity applications & 128 \\
\hline
\end{tabular}

\section{Related Work and Qualitative Comparisons to Previous Work}

To our knowledge, this paper is the first to propose and explore memory page mapping mechanisms as a solution to mitigate inter-application memory interference and thereby improve system performance.

Memory Scheduling. The problem of mitigating interference has been extensively addressed using application-aware memory request scheduling. We briefly describe the two approaches we compare our mechanisms to in Section 9. ATLAS [10] is a memory scheduling algorithm that improves system throughput by prioritizing applications based on their attained memory service. Applications that have smaller attained memory service are prioritized over others because such threads are more likely to return to long compute periods and keep their cores utilized. Thread cluster memory scheduling (TCM) [11] improves both system performance and fairness. System performance is improved by allocating a share of the main memory bandwidth for latency-sensitive applications. Fairness is achieved by shuffling scheduling priorities of memory-intensive applications at regular intervals to prevent starvation of any application. These works and other application-aware memory scheduler works $[15,16,17,19,21]$ attempt to reduce inter-application memory interference purely through memory scheduling. As a result, they require significant modifications to the memory controller's design. In contrast, we propose 1) an alternative approach to reduce memory interference which does not require changes to the scheduling algorithm when employed alone, 2) combining our channel partitioning mechanism with memory scheduling to gain better performance than either can achieve alone. Our quantitative comparisons in Section 9 show that our proposed mechanisms perform better than the current state-of-the-art scheduling policies, with no change or minimal changes to the memory scheduling algorithm.

Application-unaware memory schedulers $[9,18,22,30]$, including the commonly-employed FRFCFS policy [22, 30], aim to maximize DRAM throughput, and therefore, lead to low system performance in multi-core systems, as shown in previous work [10, 11, 15, 16, 17, 19].

OS Thread Scheduling. Zhuravlev et al. [29] aims to mitigate shared resource contention between threads by co-scheduling threads that interact well with each other on cores sharing the resource, similar to [23]. Such solutions require enough threads with symbiotic characteristics to exist in the OS's thread scheduling pool. In contrast, our proposal can reduce memory interference even if threads that interfere significantly with each other are co-scheduled in different cores and can be combined with co-scheduling proposals to further improve system performance.

Page Allocation. Page allocation mechanisms have been explored previously. Awasthi et al. [3] use page allocation/migration to balance load across memory controllers (MCs) in an application-unaware manner, to improve memory bandwidth utilization and system performance in a network-on-chip based system where a core has different distances to different memory channels. Our proposal, in comparison, performs page allocation in an application-aware manner with the aim of reducing interference between different applications. We compare our approach to an adaptation of [3] to crossbar-based multicore systems where all memory controllers are equidistant to any core (in Section 9.3) and show 
that application-aware channel partitioning leads to better system performance than balancing load in MCs. However, concepts from both approaches can be combined for further performance benefits. In NUMA-based multiprocessor systems with local and remote memories, page allocation mechanisms were used to place data close to corresponding computation node $[5,26]$. Our goal is completely different: to map data to different channels to mitigate interference between different applications. In fact, our schemes do not require the system to have non-uniform access characteristics to MCs.

Sudan et al. [24] propose to colocate frequently used chunks of data into rows, thereby improving row-buffer locality, by modifying OS page mapping mechanisms. Lebeck et al. and Hur et al. [9, 12] propose page allocation mechanisms to increase idleness and thus decrease energy consumption in DRAM ranks/banks. Phadke et al. [20] propose a heterogeneous memory system where each memory channel is optimized for latency, bandwidth, or power and propose page mapping mechanisms to map appropriate applications' data to appropriate channels to improve performance and energy efficiency. None of these works consider using page allocation to reduce inter-application memory interference, and therefore they can be potentially combined with our proposal to achieve multiple different goals.

\section{Evaluation Methodology}

Simulation Setup. MCP requires the MPKI and RBH values to be collected for each application. These per-application hardware counters, though easy to implement, are not present in existing systems. Also, our evaluation requires different system configurations with varying architectural parameters and comparison to new scheduling algorithms. For these reasons, we are unable to evaluate MCP on a real system and use an in-house cycle-level x86 multi-core simulator. The front end of the simulator is based on Pin [13]. This simulator models the memory subsystem of a CMP in detail. It enforces channel, rank, bank, port and bus conflicts, thereby capturing all the bandwidth limitations and modeling both channel and bank-level parallelism accurately. The memory model is based on DDR2 timing parameters [14], verified using DRAMSim [27]. We model the execution in a core, including the instruction-window. Unless mentioned otherwise, we model a 24-core system with 4 memory channels/controllers. Table 2 shows major processor and memory parameters.

\begin{tabular}{|c|c|}
\hline Processor Pipeline & 128-entry instruction window (64-entry issue queue, 64-entry store queue), 12-stage pipeline \\
\hline Fetch/Exec/Commit Width & 3 instructions per cycle in each core; 1 can be a memory operation \\
\hline L1 Caches & 32 K-byte per-core, 4-way set associative, 32-byte block size, 2-cycle latency \\
\hline L2 Caches & 512 K-byte per core, 8 -way set associative, 32 -byte block size, 12 -cycle latency \\
\hline DRAM controller (on-chip) & 128-entry request buffer, 64-entry write buffer, reads prioritized over writes, row interleaving \\
\hline DRAM chip parameters & DDR2-800 timing parameters, $t_{C L}=15 \mathrm{~ns}, t_{R C D}=15 \mathrm{~ns}, t_{R P}=15 \mathrm{~ns}, \mathrm{BL} / 2=10 \mathrm{~ns}, 8$ banks, $4 \mathrm{~K}$ row-buffer \\
\hline DIMM Configuration & Single-rank, 8 DRAM chips put together on a DIMM to provide a 64-bit wide memory channel \\
\hline Round-trip L2 miss latency & $\begin{array}{c}\text { For a 32-byte cache line } \\
\text { uncontended: row-buffer hit: } 40 \mathrm{~ns} \text { ( } 200 \text { cycles), closed: } 60 \mathrm{~ns} \text { ( } 300 \text { cycles), conflict: } 80 \mathrm{~ns} \text { ( } 400 \text { cycles) }\end{array}$ \\
\hline Cores and DRAM controllers & 24 cores, 4 independent DRAM controllers, each controlling a single memory channel \\
\hline
\end{tabular}

Table 2. Default processor and memory subsystem configuration.

Evaluation Metrics. We measure the overall throughput of the system using weighted speedup [23].

We also report harmonic speedup, which is a combined measure of performance and fairness.

$$
\begin{gathered}
\text { SystemThroughput }=\text { WeightedSpeedup }=\Sigma_{i} \frac{I P C_{i}^{\text {shared }}}{I P C_{i}^{\text {alone }}} \\
\text { HarmonicSpeedup }=\Sigma_{i} \frac{N}{\frac{I P C_{i}^{\text {alone }}}{I P C_{i}^{\text {shared }}}} .
\end{gathered}
$$

Our normalized results are normalized to the FR-FCFS baseline, unless stated otherwise.

Workloads. We use workloads constructed from the SPEC CPU2006 benchmarks [1] in our evaluations. 
We compiled the benchmarks using gcc with the O3 optimization flag. Table 3 shows benchmarks' characteristics. We classify benchmarks into two categories: high memory-intensity (greater than $10 \mathrm{MPKI}$ ) and low memory-intensity (less than $10 \mathrm{MPKI}$ ). We vary the fraction of high memory-intensity benchmarks in our workloads from $0 \%, 25 \%, 50 \%, 75 \%, 100 \%$ and construct 40 workloads in each category. Within each memory-intensity category, we vary the fraction of high row-buffer hit rate benchmarks in a workload from low to high. We also create another category, $\operatorname{VeryLow}(V L)$ of 40 workloads. All benchmarks in these workloads have less than 1 MPKI. We consider $V L$ for completeness, although these workloads have little bandwidth demand. For our main evaluations and some analyses, we use all 240 workloads and run each workload for 300M cycles. For sensitivity studies, we use the 40 balanced (50\% memory-intensive) workloads, unless otherwise mentioned, and run for $100 \mathrm{M}$ cycles to reduce simulation time.

\begin{tabular}{|c|c|c|c|c|c|c|c|c|c|c|c|}
\hline No. & Benchmark & MPKI & $\overline{\text { RBH }}$ & No. & Benchmark & MPKI & RBH & No. & Benchmark & MPKI & RBH \\
\hline$\overline{1}$ & 453.povray & $\overline{0.03}$ & $85.2 \%$ & 10 & 445.gobmk & 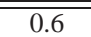 & $71 \%$ & 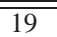 & 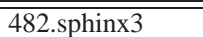 & 24.9 & (85.4\% \\
\hline 2 & 400.perlbench & 0.13 & $83.6 \%$ & 11 & 435.gromacs & 0.7 & $84.4 \%$ & 20 & 459.GemsFDTD & 25.3 & $28.8 \%$ \\
\hline 3 & 465.tonto & 0.16 & $91 \%$ & 12 & 464.h264 & 2.7 & $92.3 \%$ & 21 & 433.milc & 34.3 & $93.2 \%$ \\
\hline 4 & 454.calculix & 0.20 & $87.2 \%$ & 13 & 401.bzip2 & 3.9 & $53.8 \%$ & 22 & $470.1 \mathrm{bm}$ & 43.5 & $95.2 \%$ \\
\hline 5 & 444.namd & 0.3 & $95.4 \%$ & 14 & 456.hmmer & 5.7 & $35.5 \%$ & 23 & 462.libquantum & 50 & $99.2 \%$ \\
\hline 6 & 481.wrf & 0.3 & $91.9 \%$ & 15 & 473.astar & 9.2 & $76.2 \%$ & 24 & 450.soplex & 50.1 & $91.3 \%$ \\
\hline 7 & 403.gcc & 0.4 & $73.2 \%$ & 16 & 436.cactusADM & 9.4 & $18 \%$ & 25 & 437.1eslie3d & 59 & $82.6 \%$ \\
\hline 8 & 458.sjeng & 0.4 & $11.5 \%$ & 17 & 471.omnetpp & 21.6 & $46 \%$ & 26 & 429.mcf & 99.8 & $42.9 \%$ \\
\hline 9 & 447.dealIII & 0.5 & $81.2 \%$ & 18 & 483.xalancbmk & 23.9 & $73.2 \%$ & & & & \\
\hline
\end{tabular}

Table 3. SPEC CPU2006 benchmark characteristics.

Parameter Values. The default MPKI scaling factor and $R B H_{t}$ values we use in our experiments are 1 and 50\% respectively. For the profile interval and execution interval, we use values of 10 million and 100 million, respectively. We later study sensitivity to all these parameters.

\section{Results}

We first present and analyze the performance of MCP and IMPS on a 24-core 4-memory controller system. Figure 6 shows the system throughput and harmonic speedup averaged over all 240 workloads. The upper right part of the graph corresponds to better system throughput and a better balance between fairness and performance. MCP improves system throughput by $7.1 \%$ and harmonic speedup by $11 \%$ over the baseline. IMPS provides $4 \%$ better system throughput (13\% better harmonic speedup) over $\mathrm{MCP}$, and $11 \%$ better system throughput (24\% better harmonic speedup) over the baseline. We observe (not shown) that the scheduling component of IMPS alone (without partitioning) gains

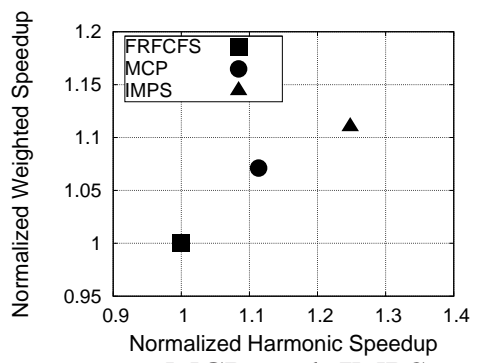

Figure 6. MCP and IMPS performance (normalized) across 240 workloads. half of the performance improvement of IMPS. We conclude that interference-aware channel partitioning is beneficial for system performance, but dividing the task of interference reduction using both channel partitioning and memory scheduling together provides better system performance than employing either alone.

Individual Workloads. Figure 7 shows the weighted speedup for four randomly selected, representative workloads shown in Table 4. We observe that MCP and IMPS gain performance benefits consistently across different workloads. 
SAFARI Technical Report No. 2011-002 (June 3, 2011)

\begin{tabular}{|c|l|l|}
\hline Workload & High memory-intensity benchmarks & Low Memory-intensity benchmarks \\
\hline \hline W1 & $\begin{array}{l}\text { perlbench, gobmk, gromacs, gcc(2), sjeng(2), } \\
\text { hmmer(2), bzip2, cactus, h264ref }\end{array}$ & $\begin{array}{l}\text { sphinx3, soplex, libquantum(4), } \\
\text { milc(3), lbm(3) }\end{array}$ \\
\hline W2 & $\begin{array}{l}\text { gromacs(2), h264(2), dealII(2), astar(2), } \\
\text { hmmer(2), cactusADM(2) }\end{array}$ & $\begin{array}{l}\text { milc(2), leslie3d(2)), sphinx(3), } \\
\text { gemsFDTD(3), libquantum(3) }\end{array}$ \\
\hline W3 & $\begin{array}{l}\text { namd(3), gcc(3), astar(3), } \\
\text { cactusADM(3) }\end{array}$ & $\begin{array}{l}\text { leslie3d(3), milc(3), omnetpp(3), } \\
\text { mcf(3) }\end{array}$ \\
\hline W4 & $\begin{array}{l}\text { tonto, astar, gcc, povray, hmmer, h264, } \\
\text { gromacs, perlbench, dealII, cactusADM(2), bzip2 }\end{array}$ & $\begin{array}{l}\text { aalancbmk, libquantum, lbm, sphinx3, milc } \\
\text { soplex, omnetpp(2), gemsFDTD(3), mcf }\end{array}$ \\
\hline
\end{tabular}

Table 4. Four representative workloads.

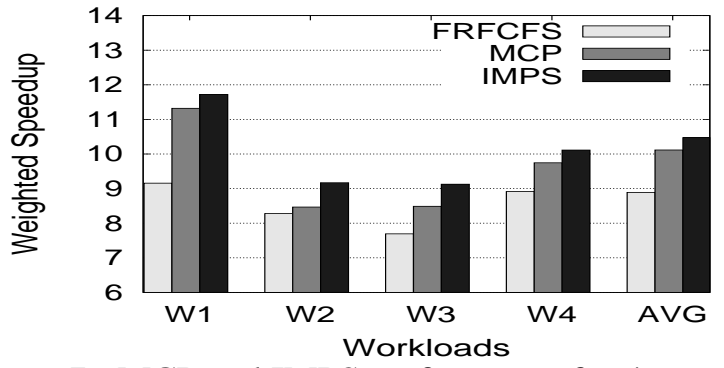

Figure 7. MCP and IMPS performance for 4 sample workloads and avg across 40 balanced workloads.

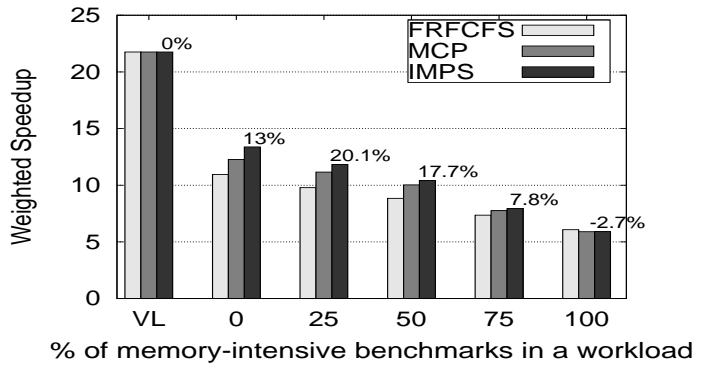

Figure 8. MCP and IMPS Performance across memory-intensity categories. \% gain values of IMPS over FRFCFS are labeled.

Effect of Workload Memory-Intensity. Figure 8 shows the system throughput benefits of MCP and IMPS, for six memory-intensity based categories of workloads. ${ }^{5}$ As expected, as workload intensity increases (from left to right in the figure), absolute system throughput decreases due to increased interference between applications.

We make three major conclusions. First, MCP and IMPS improve performance significantly over FR-FCFS in most of the memory-intensity categories. Specifically, MCP avoids interference between applications of both dissimilar and similar intensities by isolating them to different channels, enabling benefits mostly regardless of workload composition. Second, IMPS's performance benefit over MCP is especially significant in the lower-intensity workloads. Such workloads have a higher number of very low memory-intensity applications and IMPS prioritizes them in the scheduler, which is more effective for system performance than reducing interference for them by assigning them to their own channels, which wastes bandwidth as done by MCP. As the workload memory-intensity increases, IMPS' performance benefit over MCP becomes smaller because the number of low-intensity workloads becomes smaller. Third, when the workload mix is very non-intensive or very intensive, MCP/IMPS do not provide much benefit. In the $V L$ category, load on memory and as a result interference is very low, limiting the potential of MCP/IMPS. When $100 \%$ of applications in the workload are intensive, the system becomes memory bandwidth limited and conserving memory bandwidth by exploiting row-buffer locality (using simple FR-FCFS) provides better performance than reducing inter-application interference at the expense of reducing memory throughput. Any scheduling or partitioning scheme that breaks the consecutive row-buffer hits results in a system performance loss. We conclude that MCP and IMPS are effective for a wide variety of workloads where contention exists and the system is not fully bandwidth limited.

\footnotetext{
${ }^{5}$ All categories from $0-100 \%$ place a load on the memory system, as the intensity cut off used to classify an application as intensive is 10 MPKI, which is reasonably large to begin with.
} 


\subsection{Comparison with Previous Scheduling Policies}

Figure 9 compares MCP and IMPS with previous memory scheduling policies, FR-FCFS [22], PARBS [17], ATLAS [10] and TCM [11] over 240 workloads. Two major conclusions are in order. First, application-aware scheduling policies perform better than FR-FCFS, and, TCM performs the best among the application-aware scheduling policies, consistent with previous work[10, 11, 17]. Second, MCP and IMPS outperform TCM by $1 \% / 5 \%$, with no/minimal changes to the scheduler.

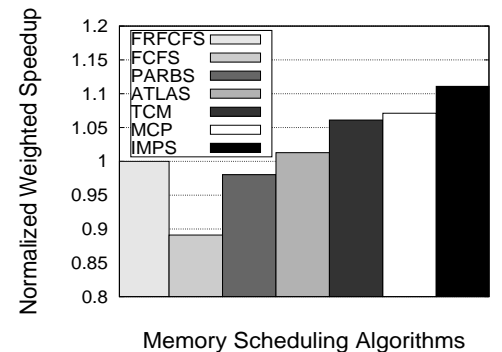

Figure 9. MCP and IMPS performance (normalized) vs previous scheduling policies averaged across 240 workloads.

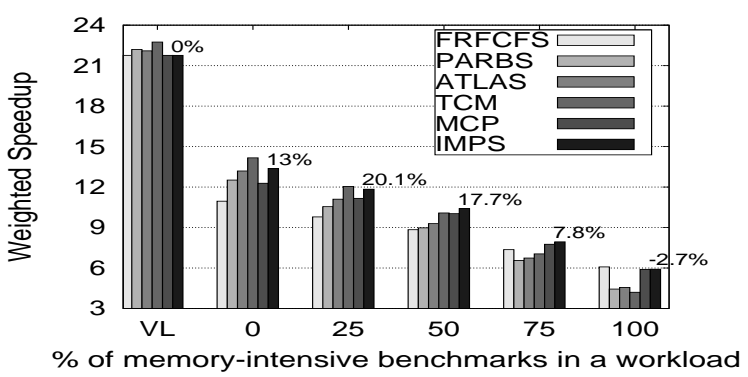

Figure 10. MCP and IMPS performance vs previous scheduling policies across memory-intensity categories. Percentage improvement values of IMPS over FR-FCFS are displayed.

Figure 10 provides insight into where MCP and IMPS' performance benefits are coming from by breaking down performance based on workload intensity. As the workload memory intensity (thus contention) increases, MCP and IMPS become more effective than pure memory scheduling approaches. At low-intensity workloads (VL, 0\%, 25\%), TCM performs slightly better than IMPS because TCM is able to distinguish and prioritize between each individual application in the memory scheduler (not true for MCP/IMPS), leading to reduced interference between low and medium intensity applications. At higher intensity workloads $(50 \%, 75 \%, 100 \%)$, reducing interference via channel partitioning is more effective than memory scheduling: both MCP and IMPS outperform TCM, e.g. by $40 \%$ in the $100 \%$-intensity workloads. In such workloads, contention for memory is very high as many high-intensity applications contend. Channel partitioning completely eliminates interference between some applications by separating out their access streams to different channels, thereby reducing the number of applications that contend with each other. On the other hand, TCM or a pure memory scheduling scheme tries to handle contention between high-intensity workloads purely by prioritization, which is more effective at balancing interference but cannot eliminate interference as MCP/IMPS does since all applications contend with each other. We conclude that IMPS is a more effective solution than pure memory scheduling especially when workload intensity (i.e., memory load) is high, which is the expected trend in future systems.

Note that IMPS's performance benefits over application-aware memory schedulers come at a significantly reduced complexity, as described in Section 6.

\subsection{Interaction with Previous Scheduling Policies}

Figure 11 compares MCP and IMPS, when implemented on top of FR-FCFS, ATLAS and TCM as the underlying scheduling policy. When IMPS is implemented over ATLAS and TCM, it adds another priority level on top of the scheduling policy's priority levels: very-low-intensity applications are prioritized over others and the scheduling policy's priorities are used between very-low-intensity applications and between the remaining applications. 
Several conclusions are in order. First, adding MCP/IMPS on top of any previous scheduling policy improves performance (IMPS gains 7\% and 3\% over ATLAS and TCM respectively), showing that our proposal is orthogonal to the underlying memory scheduling algorithm. Second, MCP/IMPS over FR-FCFS (our default proposal) provides better performance than MCP/IMPS employed over TCM or ATLAS. This is due to two reasons: 1) channel partitioning decisions MCP makes are designed assuming an FR-FCFS policy and not designed to take into account or interact well with ATLAS/TCM's more

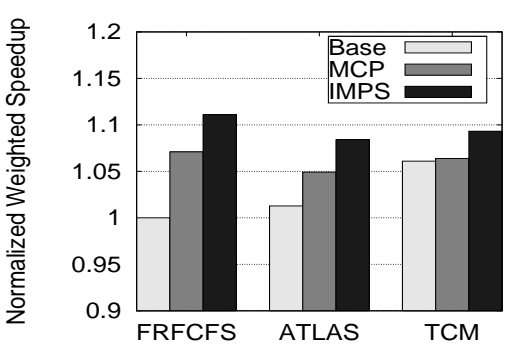

Figure 11. MCP and IMPS performance over different scheduling policies (240 workloads). sophisticated thread ranking decisions. There is room for improvement if we design a channel partitioning scheme that is specialized for the underlying scheduling policy. We leave this for future work. 2) MCP/IMPS isolates groups of similar applications to different channels and ATLAS/TCM operate within each channel to prioritize between/cluster these similar applications. However, ATLAS and TCM are designed to exploit heterogeneity between applications and do not perform as well when the applications they prioritize between are similar. We found that prioritizing similar-intensity applications over each other in the way ATLAS/TCM does, creates significant slowdowns because the applications are treated very differently. We conclude that MCP/IMPS can be employed on top of any underlying scheduler to gain better performance over using the scheduler alone. However, it performs best when employed over an FR-FCFS baseline for which it is designed.

\subsection{Comparison with Prior Work on Balancing Load Across Multiple Memory Controllers}

In [3], Awasthi et al propose two page allocation schemes to balance the load across multiple memory controllers: 1) page allocation on first touch (Adaptive First Touch, AFT), 2) Dynamic Page Migration (DPM). AFT attempts to balance load by allocating a page to a channel which has the minimum value of a cost function involving channel load, row buffer hit rate, and, the distance to the channel. DPM proposes to migrate a certain number of pages from the channel with the highest load to the least loaded channel at regular intervals, in addition to AFT. In our adaptation of AFT, we consider both channel load and row-buffer-hit rate but do not incorporate the channel distance, as we do not model a network-on-chip. Figure 12 compares MCP/IMPS performance to that of AFT and DPM. First, AFT and DPM both improve performance by $5 \%$ over the baseline, because they reduce memory access latency by balancing load across different channels. The gains from the two schemes are similar as the access patterns of the applications we evaluate do not vary largely with time, resulting in very few invocations of dynamic page migration. Second, our proposals outperform AFT and DPM by 7\% (MCP) and $12.4 \%$ (IMPS), as they proactively reduce inter-application interference by using application characteristics, while AFT and DPM are not interference- or application-aware and try to reactively balance load across memory controllers. We conclude that reducing inter-application interference by page allocation provides better performance than balancing load across memory controllers in an application-unaware manner. 


\subsection{Impact of Cache Line Interleaving}

We study the effect of MCP/IMPS on a system with a restricted form of cache line interleaving that maps consecutive cache lines of a page across banks within a channel. Figure 13 shows that MCP/IMPS improve the performance of such a system by $5.1 \%$ and $11 \%$ respectively. We observed (not shown) that unrestricted cache line interleaving across channels (to which MCP/IMPS cannot be applied) improves performance by only $2 \%$ over restricted cache line interleaving. Hence, using channel partitioning with MCP/IMPS outperforms cache line interleaving across channels. This is because the reduction in interapplication interference with MCP/IMPS provides more system performance benefit than the increase of channel-level parallelism with unrestricted cache-line interleaving. We conclude that MCP/IMPS are effective independent of the interleaving policy employed, as long as the interleaving policy allows the mapping of an entire page to a channel (which is required for MCP/IMPS to be implementable).

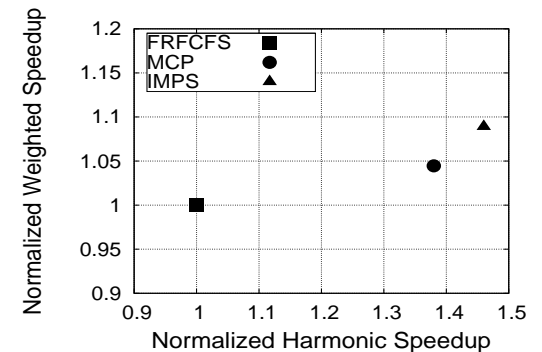

Figure 13. System throughput and harmonic speedup with cache line interleaving (240 workloads).

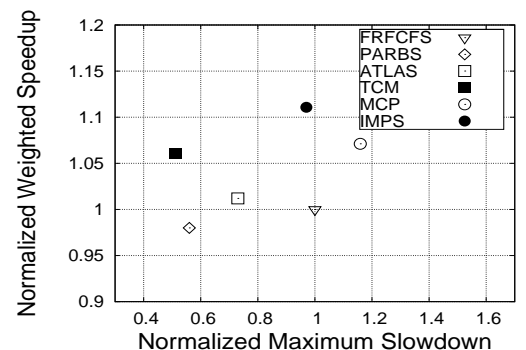

Figure 14. Performance and fairness compared to previous scheduling policies (240 workloads).

\subsection{Effect of MCP and IMPS on Fairness}

The fairness metric we use, the maximum slowdown of a workload, is defined as the maximum of the slowdowns (inverse of speedups) of all applications [10, 11, 25]; lower maximum slowdown values are more desirable. Figure 14 shows throughput vs fairness of previously proposed scheduling policies and our proposed schemes. IMPS has slightly better fairness (3\% lower maximum slowdown) than FR-FCFS. While MCP and IMPS provide the best performance compared to any other previous proposal, they result in higher unfairness. Note that this is expected by design: MCP and IMPS are designed for improving system performance and not fairness. They make the conscious choice of placing high-intensity (and high-row-locality) applications onto the same channel(s) to enable faster progress of lower-intensity applications, which sometimes results in the increased slowdown of higher-intensity applications. Channel partitioning based techniques that can improve both performance and fairness are out of the scope of the paper and an interesting area of future work.

\subsection{Sensitivity Studies}

Sensitivity to MCP and IMPS algorithm parameters. We first vary the profile interval length to study its impact on MCP and IMPS' performance (Figure 15). A shorter initial profile interval length (1 and 5 Million) leads to less stable MPKI and RBH values, leading to inaccurate estimation of application characteristics. In contrast, a longer profile interval length causes a number of pages to be allocated prior to computing channel preferences. A profile interval length of $10 \mathrm{M}$ cycles balances these downsides of shorter and larger intervals and provides the best performance. We also experimented with 
different execution interval lengths (Figure 16). A shorter interval leads to better adaptation to changes in application behavior but also higher overhead due to page migration if application characteristics are not stable within the interval. A longer interval might miss changes in the behavior of applications. A $100 \mathrm{M}$-cycle interval ensures a good balance and provides good performance.

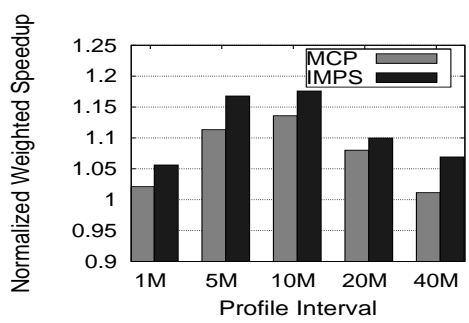

Figure 15. Performance vs Profile interval (40 workloads).

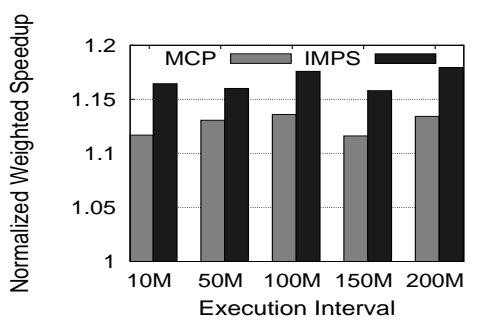

Figure 16. Performance vs Execu- Figure

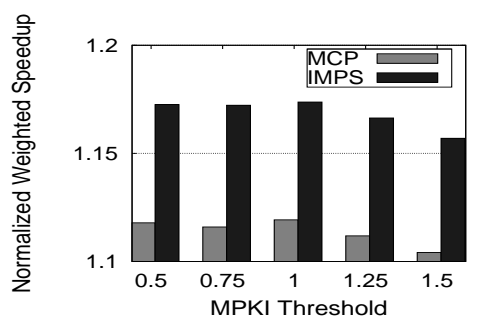
$M P K I_{t}$ (40 workloads).

Figure 17 shows the sensitivity of MCP/IMPS to $M P K I_{t}$. As $M P K I_{t}$ is increased beyond 1, more medium and high memory-intensity applications get into the low memory-intensity group, thereby slowing down the low-intensity applications and resulting in lower throughput. We also varied $R B H_{t}$, the row buffer-hit rate threshold and the very low memory-intensity threshold. System performance remains high and stable over a wide range of these values, with the best performance observed at an $R B H_{t}$ value of $50 \%$ and a very low memory-intensity threshold value of 1.5 .

Scalability to cores, MCs and cache sizes. Table 5 shows the performance of IMPS as number of cores, number of MCs and L2 cache size are varied. The rest of the system remains the same. IMPS' benefits are significant across all configurations. IMPS' performance gain in general increases when the system is more bandwidth constrained, i.e., with increasing number of cores and reducing number of MCs. MCP shows similar trends as IMPS.

\begin{tabular}{|c||c|c|c||c|c|c||c|c|c|}
\hline \multicolumn{1}{|c||}{} & \multicolumn{3}{c||}{ No. of Cores } & \multicolumn{3}{c||}{ No. of MCs } & \multicolumn{3}{c|}{ Private L2 Cache Size } \\
\hline \hline & 16 & 24 & 32 & 2 & 4 & 8 & $256 \mathrm{~KB}$ & $512 \mathrm{~KB}$ & $1 \mathrm{MB}$ \\
\hline IMPS System Throughput Improvement & $15.8 \%$ & $17.4 \%$ & $31 \%$ & $18.2 \%$ & $17.1 \%$ & $10.7 \%$ & $16.6 \%$ & $17.4 \%$ & $14.3 \%$ \\
\hline
\end{tabular}

Table 5. Sensitivity to number of cores, number of MCs, and L2 cache size (40 workloads).

\section{Conclusion}

We presented 1) MCP, a fundamentally new approach to reducing inter-application interference at the memory system, by mapping the data of interfering applications to separate channels, 2) IMPS, that effectively divides the work of reducing inter-application interference between the system software and the memory scheduler. Our extensive qualitative and quantitative comparisons demonstrate that MCP and IMPS both provide better system performance than the state-of-the-art memory scheduling policies, with no or minimal hardware complexity. IMPS provides better performance than channel partitioning or memory scheduling alone. We conclude that inter-application memory interference is best reduced using the right combination of page allocation to channels and memory scheduling, and that IMPS achieves this synergy with minimal hardware complexity. 


\section{Acknowledgements}

We gratefully acknowledge Yoongu Kim, members of the SAFARI research group and CALCM at CMU and the Microsystems Design Lab at PSU for many insightful discussions on this work. We acknowledge the support of our industrial sponsors; AMD, Intel, and Microsoft. This research was partially supported by grants from the Gigascale Systems Research Center, the National Science Foundation (CAREER Award CCF-0953246), and Carnegie Mellon CyLab.

\section{References}

[1] SPEC CPU2006. http://www.spec.org/spec2006. 5, 12

[2] The AMD processor roadmap for industry standard servers, 2010. 2

[3] M. Awasthi et al. Handling the problems and opportunities posed by multiple on-chip memory controllers. In PACT-19, 2010. 9, 11, 16

[4] J. Casazza. First the tick, now the tock: Next generation intel microarchitecture (Nehalem). In Intel White Paper, 2009. 2

[5] R. Chandra et al. Scheduling and page migration for multiprocessor compute servers. In ASPLOS, 1994. 12

[6] V. Cuppu et al. A performance comparison of contemporary DRAM architectures. In ISCA-26, 1999. 3

[7] R. Das et al. Application-to-core mapping policies to reduce interference in on-chip networks. In SAFARI Technical Report No. 2011-001, 2011. 9

[8] E. Ebrahimi et al. Fairness via source throttling: a configurable and high-performance fairness substrate for multi-core memory systems. In ASPLOS-15, 2010. 7

[9] I. Hur and C. Lin. Adaptive history-based memory schedulers. In MICRO-37, 2004. 11, 12

[10] Y. Kim et al. ATLAS: A scalable and high-performance scheduling algorithm for multiple memory controllers. In HPCA-16, 2010. 2, 3, 9, 10, 11, 15, 17

[11] Y. Kim et al. Thread cluster memory scheduling: Exploiting differences in memory access behavior. In MICRO-43, 2010. 2, 3, 7, 9, 10, 11, 15, 17

[12] A. Lebeck et al. Power aware page allocation. In ASPLOS-9, 2000. 12

[13] C. Luk et al. Pin: building customized program analysis tools with dynamic instrumentation. In PLDI, 2005. 12

[14] Micron. IGb DDR2 SDRAM Component: MT47H128M8HQ-25. 12

[15] T. Moscibroda and O. Mutlu. Memory performance attacks: Denial of memory service in multi-core systems. In USENIX Security, 2007. 2, 11

[16] O. Mutlu and T. Moscibroda. Stall-time fair memory access scheduling for chip multiprocessors. In MICRO40, 2007. 2, 3, 7, 9, 11

[17] O. Mutlu and T. Moscibroda. Parallelism-aware batch scheduling: Enhancing both performance and fairness of shared DRAM systems. In ISCA-35, 2008. 2, 9, 10, 11, 15

[18] C. Natarajan et al. A study of performance impact of memory controller features in multi-processor server environment. In WMPI-3, 2004. 11

[19] K. Nesbit et al. Fair queuing memory systems. In MICRO-39, 2006. 2, 11

[20] S. Phadke and S. Narayanasamy. MLP aware heterogeneous memory system. In DATE, 2011. 12

[21] N. Rafique et al. Effective management of DRAM bandwidth in multicore processors. In PACT, 2007. 2,11 
[22] S. Rixner et al. Memory access scheduling. In ISCA-27, 2000. 3, 11, 15

[23] A. Snavely and D. M. Tullsen. Symbiotic jobscheduling for a simultaneous multithreaded processor. In ASPLOS-9, 2000. 11, 12

[24] K. Sudan et al. Micro-pages: increasing DRAM efficiency with locality-aware data placement. In ASPLOS15, 2010. 12

[25] H. Vandierendonck and A. Seznec. Fairness metrics for multi-threaded processors. Computer Architecture Letters, PP(99):1, 2011. 17

[26] B. Verghese et al. Operating system support for improving data locality on cc-numa compute servers. In ASPLOS-7, 1996. 12

[27] D. Wang et al. DRAMsim: a memory system simulator. In SIGARCH Comp. Arch. News. 33(4), 2005. 12

[28] G. L. Yuan et al. Complexity effective memory access scheduling for many-core accelerator architectures. In MICRO-42, 2009. 2

[29] S. Zhuravlev et al. Addressing shared resource contention in multicore processors via scheduling. In ASPLOS-15, 2010. 11

[30] W. Zuravleff and T. Robinson. Controller for a synchronous DRAM that maximizes throughput by allowing memory requests and commands to be issued out of order. 1997. 3, 11 